\title{
Performance Analysis of DCT and WDCT Algorithms in Image Steganography
}

Graduate student,

Al Anood Najeeb, Noor Al Maadeed, Somaya Al Maadeed

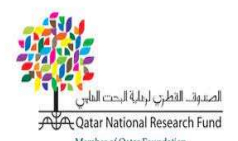

\section{Information and Communication Technologies}

(ICT)

\section{Introduction}

Steganography is the process of embedding covert message inside the cover to transfer the confidential messages from the sender to the receiver. Various steganography techniques have been developed to date, and they utilize various digital formats such as text, photos, audio, and video.

Common method used in image steganography is the Least significant bit method (LSB). Even though LSB has high capacity, they are not robust against compression and not secure against steganalysis [1].

To overcome this, image steganographic methods in frequency domain such as Discrete Cosine Transform (DCT) and the extended version of DCT i.e., Warped Discrete Cosine Transform (WDCT) [4] is introduced. These methods ensure high robustness and imperceptibility compared to LSB.

The main aim of this work is to evaluate the performance of DCT and WDCT on different public datasets.

\section{General Steps in Image Steganography}

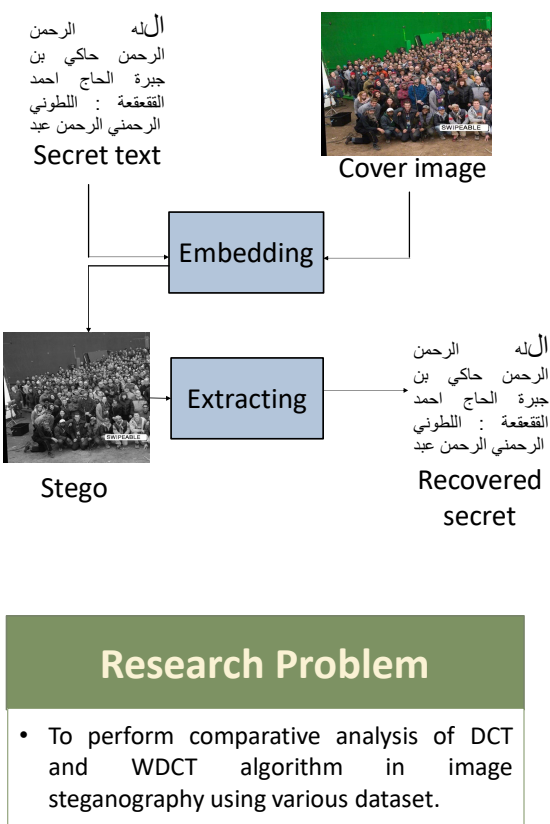

\section{Methodology}

In this methodology, the stego image is converted from spatial domain to frequency domain.

- Embedding : The cover image used for hiding the secret is divided into multiple $8 \times 8$ blocks of pixel. In each of the pixel, 2D DCT is applied. The DCT coefficient is quantized using the quantization matrix. The secret data is preprocessed to produce the secret bits and the secret is embedded inside the DCT coefficients. After embedding, the stego image is obtained.

- Extraction : Apply inverse 2D DCT to the modified block of pixels and generate the recovered secret.

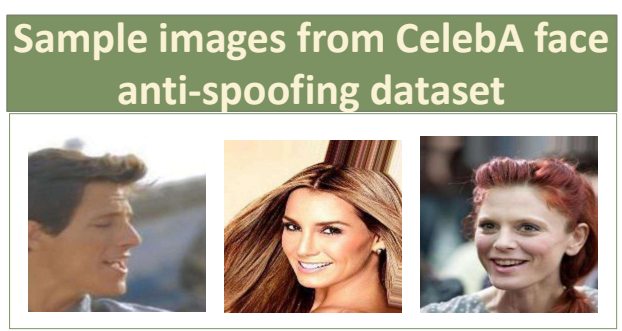

Sample images from NWPUCrowd dataset
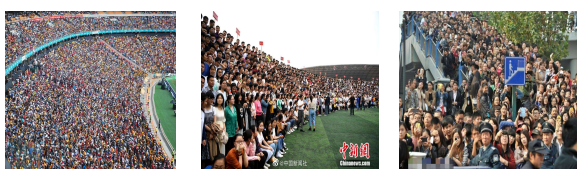

\section{Experimental Results}

\begin{tabular}{|l|l|c|c|c|c|}
\hline Dataset & Method & $\begin{array}{c}\text { PSNR } \\
\text { (db) }\end{array}$ & $\begin{array}{c}\text { Encode } \\
\text { Time (s) }\end{array}$ & $\begin{array}{c}\text { Decode } \\
\text { Time } \\
\text { (s) }\end{array}$ & $\begin{array}{c}\text { Length } \\
\text { (bytes) }\end{array}$ \\
\hline $\begin{array}{c}\text { NWPU- } \\
\text { Crowd } \\
{[2]}\end{array}$ & DCT & 38.59 & 1.75 & 0.77 & 349 \\
\cline { 2 - 6 } & WDCT & 40.55 & 0.53 & 0.24 & 349 \\
\hline $\begin{array}{c}\text { CelebA } \\
\text { face } \\
\text { anti- } \\
\text { spoofing } \\
{[3]}\end{array}$ & DCT & 40.37 & 0.24 & 0.11 & 349 \\
\hline
\end{tabular}

[3]

\section{Image Results}
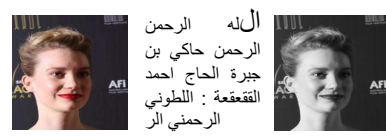

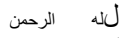

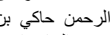

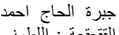
الرحمني الزي

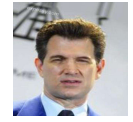

1

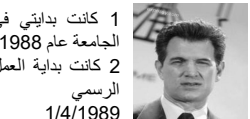

1 كانت بدايتي في 1988 الجمانت بداية العطل الرسمي

cover image

secret text

stego image

recovered secret

Resultant Images of CelebA face anti-spoofing dataset against DCT and WDCT algorithm
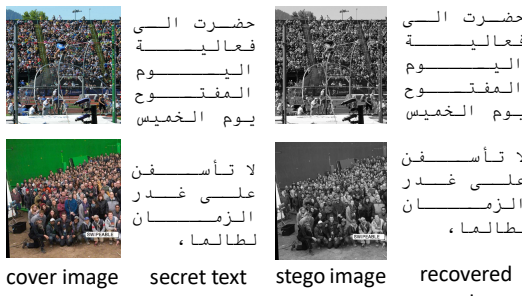

cover image

secret text

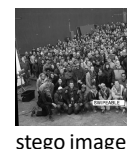

secret

Resultant Images of NWPU-Crowd dataset against DCT and WDCT algorithm

\section{Discussion and Conclusion}

It is evident from the experimental results that the performance of the frequency domain methods such as DCT and WDCT on different public datasets has achieved higher accuracy. The PSNR value, encoding time and decoding time is calculated for each experiments.

The resultant images proves that the methods are very less prone to noise attack..

Among the algorithms, WDCT has high imperceptibility and robustness with minimal or no distortion with high PSNR metrics.

Therefore, these image steganographic technique produces high security compared to traditional method namely LSB.

The privacy of differential confidential data can be secured through image steganography.

\section{Acknowledgment}

This work was supported by the Qatar National Research Fund (a member of Qatar Foundation) under Grant NPRP11S-0113180276. Open Access funding provided by the Qatar National Library.

\section{Contact}

Al Anood Najeeb

Department of computer science and engineering

Email:an1912824@qu.edu.qa

\section{References}

Dalal, Mukesh \& Juneja, Mamta. (2021). A survey on information hiding using video steganography Artificial Intelligence Review. 65. 10.1007/s10462-021-09968-0.

. Wang, Q., Gao, J., Lin, W. and Li, X., 2020. NWPU-crowd: A large-scale benchmark for crowd counting and localization. IEEE transactions on pattern analysis and machine intelligence, 43(6), pp.2141-2149.

Y. Zhang, Z. Yin, Y. Li, G. Yin, J. Yan, J. Shao, and Z. Liu, "Celeba-spoof: Large-scale face anti-spoofing dataset with rich annotations," in European Conference on Computer Vision. Springer, 2020, pp. 70-85.

N. I. Cho and S. K. Mitra, "Warped discrete cosine transform and its application in image compression," IEEE Transactions on Circuits and Systems for Video Technology, vol. 10, no. 8, pp. 1364-1373, 2000. 